\title{
Decolonization of Staphylococcus aureus in patients with atopic dermatitis: a reason for increasing resistance to antibiotics?
}

\author{
Izabela Błażewicz' ${ }^{1}$ Maciej Jaśkiewicz², Marta Bauer², Lidia Piechowicz³ ${ }^{3}$ Roman J. Nowicki' ${ }^{1}$, Wojciech Kamysz ${ }^{2}$, \\ Wioletta Barańska-Rybak ${ }^{1}$
}

\begin{abstract}
'Department of Dermatology, Venereology and Allergology, Medical University of Gdansk, Gdansk, Poland 2Department of Inorganic Chemistry, Faculty of Pharmacy, Medical University of Gdansk, Gdansk, Poland ${ }^{3}$ Department of Medical Microbiology, Medical University of Gdansk, Gdansk, Poland
\end{abstract}

Adv Dermatol Allergol 2017; XXXIV (6): 553-560

DOI: https://doi.org/10.5114/ada.2017.72461

\begin{abstract}
Introduction: Exacerbation of atopic dermatitis can be associated with bacterial infection. The skin of patients is colonized with Staphylococcus aureus in $90 \%$ of cases. An attempt has been made to demonstrate that eradication significantly reduces the severity of the disease. Studies indicate the efficacy of topical antibiotics, topical corticosteroids and calcineurin inhibitors. Due to increasing resistance to drugs and the defective antimicrobial peptide profile, decolonization is virtually impossible.

Aim: To determine the prevalence of $S$. aureus colonization among patients with atopic dermatitis and to assess antimicrobial susceptibility of isolated strains to antibiotics, especially fusidic acid and mupirocin.

Material and methods: One hundred patients with atopic dermatitis and 50 healthy subjects were microbiologically assessed for the carriage of $S$. aureus. Antimicrobial susceptibility tests were performed using the broth-microdilution method for antibiotics: ampicillin, ciprofloxacin, daptomycin, erythromycin, fusidic acid, linezolid, lincomycin, mupirocin, tetracycline and vancomycin.

Results: Staphylococcus aureus strains were isolated from the majority of our patients, either from the skin (71\%) or the anterior nares (67\%). In the present study, $10 \%$ of isolations represented methicillin-resistant S. aureus (MRSA). Antibiotics exhibited diverse activities against clinical isolates of $S$. aureus. Among those tested, the highest rates of resistance were shown for ampicillin $-58.5 \%$, lincomycin $-37.5 \%$ and erythromycin $-31.0 \%$. Enhanced resistance levels were expressed to mupirocin (17.5\%) and fusidic acid (15.5\%).

Conclusions: According to the increasing rate of resistance and quick recolonization after discontinuation of the treatment, chronic use of topical antibiotics is not recommended and should be limited to exacerbation of atopic dermatitis with clinical signs of bacterial infection.
\end{abstract}

Key words: Staphylococcus aureus, atopic dermatitis, mupirocin, fusidic acid.

\section{Introduction}

Atopic dermatitis (AD) is a chronic, pruritic skin disease mainly affecting children, which follows a remitting and relapsing course. It occurs in $10 \%$ to $20 \%$ of children and 1 to $3 \%$ of adults [1]. Patients with AD have a unique predisposition to be colonized or infected by a number of microbial organisms, mostly Staphylococcus aureus and Herpes simplex. Studies have shown that between $80 \%$ and $100 \%$ of patients with AD present nasal or skin colonization by $S$. aureus, while the prevalence is $5 \%$ to $30 \%$ in healthy individuals [2]. A correlation between the severity of the eczema and colonization with $S$. aureus has been demonstrated, and it has been determined that bacterial colonization is an important factor aggravating skin lesions [3]. The pathogens concentration $\left(\mathrm{cfu} / \mathrm{cm}^{2}\right)$ on the skin of $A D$ patients is significantly higher than that of healthy population [4]. Suppressed levels of ceramides, free lipoid acids, superficial polar lipids, skin natural antimicrobial peptides (LL-37, $\beta$-defensin), fibronectin receptors exposure of adhesin-binding cell wall of $S$. aureus, and destruction of the skin barrier by substances excreted by these germs are responsible for skin colonization in $A D$ [5]. Worldwide

Address for correspondence: Izabela Błażewicz MD, PhD, Department of Dermatology, Venereology and Allergology, Medical University of Gdansk, Gdansk, Poland, fax: +48 791002 315, e-mail: izabela.blazewicz@wp.pl Received: 4.12.2015, accepted: 19.08.2016. 
studies suggest that the prevalence of methicillin-resistant S. aureus (MRSA) in the population with $A D$ varies from 0 to $30.8 \%$ [6-8]. In the USA, where Community AcquiredMRSA (CA-MRSA) is now the most common pathogen cultured from patients with skin and soft-tissue infections in emergency departments, the colonization rate of $A D$ patients is as high as $18.3 \%$ [9]. There are no prevalence data for MRSA colonization among AD patients in Poland.

\section{Aim}

The aim of this study was to determine the prevalence of MRSA and $S$. aureus colonization in this group of patients, and to evaluate the antibiotic resistance of the isolated strains.

\section{Material and methods}

\section{Patients and bacterial isolates}

Patients were enrolled in our study during their visits in the Outpatient Clinic and during hospitalization in the Department of Dermatology, Venereology and Allergology in Gdańsk (Medical University in Gdansk, 28 beds) from August 2014 to August 2015. There was no selection of patients by sex or by localization and severity of lesions. Atopic dermatitis was diagnosed following the criteria of Hanifin and Rajka, which include among others pruritus, typical morphology and distribution of eczematous lesions, chronicity of the disease and personal or family history of atopy [10]. The study was approved by the local Research Ethics Board (approval number NKBBN/242-477/2014). Voluntary informed consent in writing was obtained from all participants. The exclusion criteria included chronic dermatological condition with a compromised skin barrier (e.g. psoriasis), diagnosis of any other chronic condition that increases the risk of MRSA colonization, oral or intravenous antibiotic treatment in the previous 4 weeks, treatment with topical antibiotics in the past 2 weeks, treatment with systemic corticosteroids or immunosuppressive drugs in the past 4 weeks, history of hospitalization, surgery, dialysis or residence in a long-term facility in the past year, indwelling catheter or a percutaneous device at the time of enrollment. Skin and nasal swabs (Bionovo, sterile swabs with medium Amies) collected from 100 patients with $A D$ and 50 controls were used to detect the presence of S. aureus. The control group consisted of patients without personal or family history of skin or allergic diseases who visited the Dermatological Outpatient Clinic for the dermatoscopy evaluation of moles.

\section{Identification of $S$. aureus and MRSA strains}

Preliminary identification and detection of SA and MRSA strains was conducted on the ChromID MRSA/ ChromID S. aureus biplate (bioMérieux) for simultane- ous detection of $S$. aureus and methicillin-resistant S. aureus (MRSA).

\section{Antimicrobial agents}

Antimicrobial susceptibility tests were performed in case of positive SA and MRSA strains isolated. The antibiotics tested included ampicillin (Carl Roth $\mathrm{GmbH}$ ), ciprofloxacin (Fluka), daptomycin (Sigma-Aldrich), erythromycin (Sigma-Aldrich), fusidic acid (Sigma-Aldrich), linezolid (Sigma-Aldrich), lincomycin (Sigma-Aldrich), mupirocin (Sigma-Aldrich), tetracycline (Sigma-Aldrich) and vancomycin (Sigma-Aldrich).

\section{Antimicrobial activity}

Minimal inhibitory concentration (MIC) was determined manually by the broth microdilution method in Mueller Hinton broth according to the Clinical and Laboratory Standards Institute (CLSI) recommendations [11]. Assays for daptomycin were performed in a medium supplemented with $\mathrm{Ca}^{2+}(50 \mathrm{mg} / \mathrm{l})$. Polypropylene 96 -well plates with bacteria at initial inoculums of $0.5 \times 10^{5} \mathrm{CFU} /$ $\mathrm{ml}$ exposed to tested compounds were incubated for $18 \mathrm{~h}$ at $37^{\circ} \mathrm{C}$. Minimal inhibitory concentration was taken as the lowest concentration of the compound at which a visible growth of bacteria was not observed. According to CLSI and EUCAST (European Committee on Antimicrobial Susceptibility Testing) recommendations, the experiments were performed in triplicate. Research was conducted in the laboratory of the Department of Inorganic Chemistry, Medical University in Gdansk. The bacterial isolates were categorized into the resistant or susceptible ones using interpretative criteria provided by CLSI [11].

\section{Results}

\section{Patients and bacterial isolates}

A total of 200 specimens were collected from 100 patients during the study. Atopic dermatitis patients consisted of $55 \%$ of males and $45 \%$ of females, age: 1 to 63 years, median: $22.3 \pm 15.6$ years. Staphylococcus aureus was reported in 75 (75\%) of 100 skin swabs and 73 of 100 nasal swabs (73\%). Six (6\%) of 100 nasal swabs and 5 (5\%) of 100 skin swabs were positive for MRSA. A total of 100 specimens were collected from 50 healthy subjects in the control group. Seven (14\%) of 50 nasal swabs and 2 (4\%) of 50 skin swabs were positive for S. aureus. MRSA colonization was not reported in the control group. Clinical characteristics are shown in Table 1.

\section{Antibiotic sensitivity pattern of S. aureus}

The antibiotics used in this study exhibited diverse activities against clinical isolates of $S$. aureus. The vancomycin MIC value was the lowest among the tested antibiotics and varied between 0.125 and $8 \mu \mathrm{g} / \mathrm{ml}$. Minimal inhibitory concentration 90 (the minimal inhibitory con- 
centration for $90 \%$ of isolates) was the lowest for vancomycin and ciprofloxacin. The highest MIC 90 was noted for erythromycin, lincomycin, mupirocin and tetracycline among patients with $A D$. Similar results were obtained for the control group (Table 2). Among the conventional antibiotics tested, the highest rates of resistance were shown for ampicillin - 58.5\%, daptomycin - 54.7\%, lincomycin $-37.5 \%$ and erythromycin $-31.0 \%$. The following percentage of resistant strains were noted to mupirocin $-17.5 \%$, tetracycline $-15.5 \%$, and ciprofloxacin $-13.0 \%$. In the control group, strains resistant to ampicillin $-4 \%$, daptomycin $-4 \%$, erythromycin $-2 \%$, fusidic acid $-1 \%$, lincomycin $-3 \%$, and tetracycline $-1 \%$ were noticed. One hundred percent of strains in the control group were sensitive to ciprofloxacin, linezolid, mupirocin and vancomycin. The antimicrobial resistance patterns (\%) of S. aureus in patients with atopic dermatitis and control group are shown in Figure 1. For the following antibiotics: ciprofloxacin, erythromycin, lincomycin, and tetracycline, the percentage of resistant strains was significantly higher in the MRSA group as compared to MSSA (Figure 2).

\section{Discussion}

Epidemiology of $S$. aureus and MRSA strains in AD

Methicillin-resistant $S$. aureus is a major pathogen in many infectious diseases. In 1961, the first methicillinresistant strains were reported. In 1980, MRSA strains became an endemic problem at different proportions at hospitals in several countries. Commonly, infections caused by MRSA were limited to hospitals (Hospital Acquired-MRSA, HA-MRSA). Community-acquired in-
Table 1. Clinical characteristics of patients with atopic dermatitis and the control group

\begin{tabular}{lccc}
\hline Parameter & Patients & Control & $P$-value \\
\hline Total no.: & 100 & 50 & $0.0001^{\mathrm{b}}$ \\
\hline Children & 38 & 2 & \\
\hline Adults & 62 & 48 & \\
\hline Age [years] & $22.3 \pm 15.6$ & $35.1 \pm 13.4$ & $0.0001^{\mathrm{a}}$ \\
\hline Gender (\%): & & & \\
\hline Female & 45 & 50 & \\
\hline Male & 55 & 50 &
\end{tabular}

\begin{tabular}{lccc}
\hline Presence of SA (\%): & & & $0.0001^{\mathrm{b}}$ \\
\hline On the skin & 75 & 4 & \\
\hline Anterior nares & 73 & 14 \\
\hline Presence of MRSA (\%): & 11 & 0 \\
\hline CA-MRSA & 54.5 & 0 \\
\hline HA-MRSA & 45.5 & 0 \\
\hline
\end{tabular}

aU Mann-Whitney test, ${ }^{b} \chi^{2}$ test.

fections (Community Acquired-MRSA, CA-MRSA) have been increasingly recorded since the last decade. The first report on CA-MRSA infection in a patient without any contact with the hospital environment was recorded in 1980 in the United States. Worldwide studies suggest that the prevalence of MRSA in the population with AD is divergent. For example, in the study carried by Hoeger in 2004 on pediatric outpatient population with AD, the MRSA strains were not identified [12]. However, in New

Table 2. Minimal inhibitory concentration (MIC) values of Staphylococcus aureus strains isolated from atopic dermatitis (AD) patients and control group

\begin{tabular}{|c|c|c|c|c|c|c|c|}
\hline \multirow[t]{3}{*}{ Agents } & \multicolumn{6}{|c|}{$\mathrm{MIC}[\mu \mathrm{g} / \mathrm{ml}]$} & \multirow[t]{3}{*}{$P$-value } \\
\hline & \multicolumn{3}{|c|}{ Patients with AD } & \multicolumn{3}{|c|}{ Control group } & \\
\hline & Range & MIC 50 & MIC 90 & Range & MIC 50 & MIC 90 & \\
\hline Ampicillin & $0.125-32.0$ & 2.0 & 16.0 & $0.25-32.0$ & 16.0 & 32.0 & 0.125584 \\
\hline Ciprofloxacin & $0.125->512.0$ & 0.5 & 2.0 & $0.125-1.0$ & 0.25 & 1.0 & 0.2214 \\
\hline Daptomycin & $0.125-4.0$ & 2.0 & 4.0 & $1.0-2.0$ & 2.0 & 2.0 & 0.6965 \\
\hline Erythromycin & $0.125->512.0$ & 0.5 & $>512.0$ & $0.125->512.0$ & 1.0 & $>512.0$ & 0.7188 \\
\hline Fusidic acid & $0.0-32.0$ & 0.125 & 8.0 & $0.125-16.0$ & 1.0 & 16.0 & 0.0242 \\
\hline Linezolid & $0.25-32.0$ & 1.0 & 4.0 & $1.0-4.0$ & 2.0 & 4.0 & 0.125059 \\
\hline Lincomycin & $0.25->512.0$ & 1.0 & 64.0 & $0.25->512.0$ & 0.5 & $>512.0$ & 0.25239 \\
\hline Mupirocin & $0.125->512.0$ & 0.5 & $>512.0$ & $0.125-32.0$ & 0.25 & 32.0 & 0.25257 \\
\hline Tetracycline & $0.125-64.0$ & 0.5 & 32.0 & $0.125-32.0$ & 0.5 & 32.0 & 0.8630 \\
\hline Vancomycin & $0.125-8.0$ & 0.5 & 2.0 & $0.25-1.0$ & 1.0 & 1.0 & 0.8069 \\
\hline
\end{tabular}

MIC $50-$ MIC for $50 \%$ of isolates. MIC $90-$ MIC for $90 \%$ of isolates. Significant differences between groups determined with U Mann-Whitney test. 


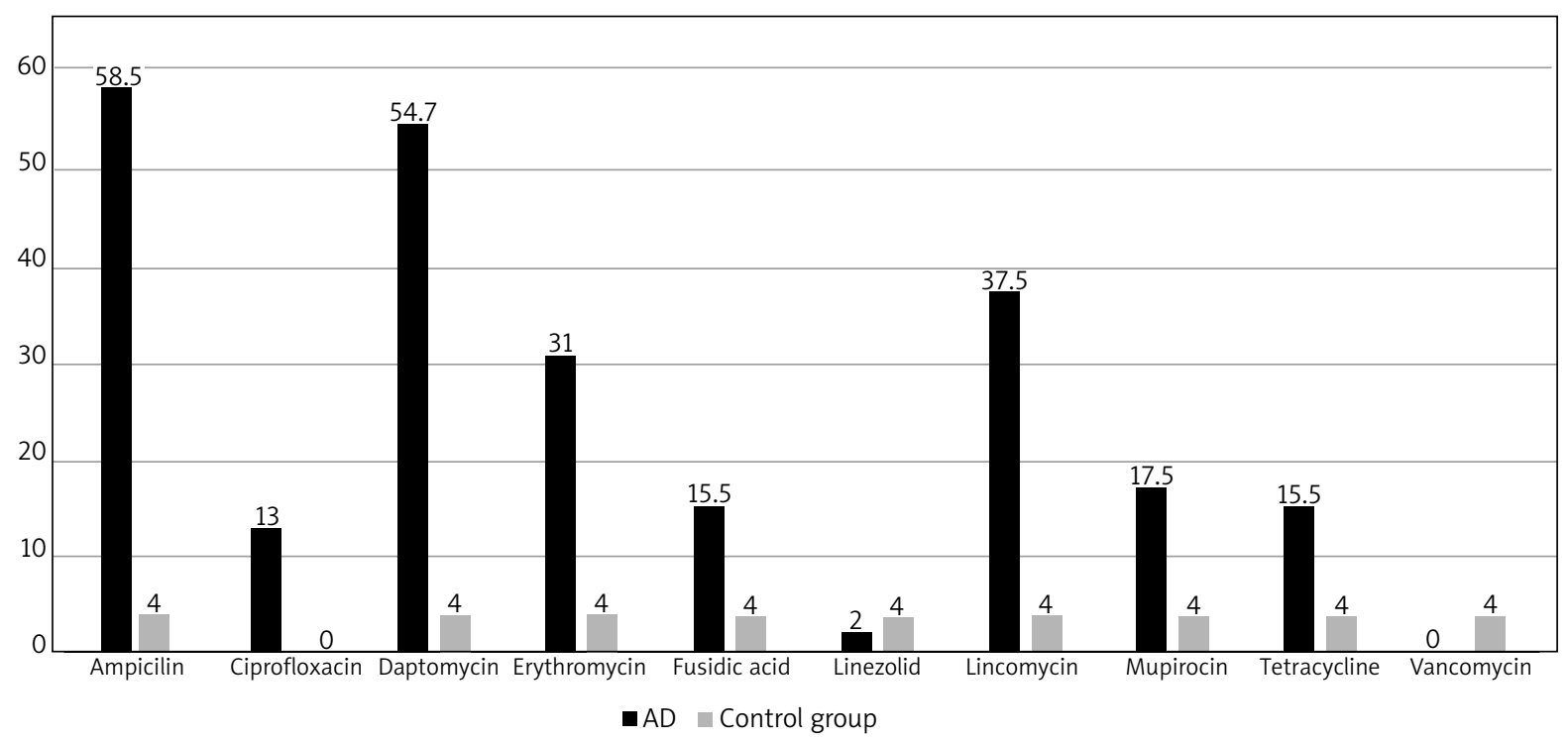

Figure 1. Antimicrobial resistance patterns (\%) of S. aureus in patients with atopic dermatitis and the control group

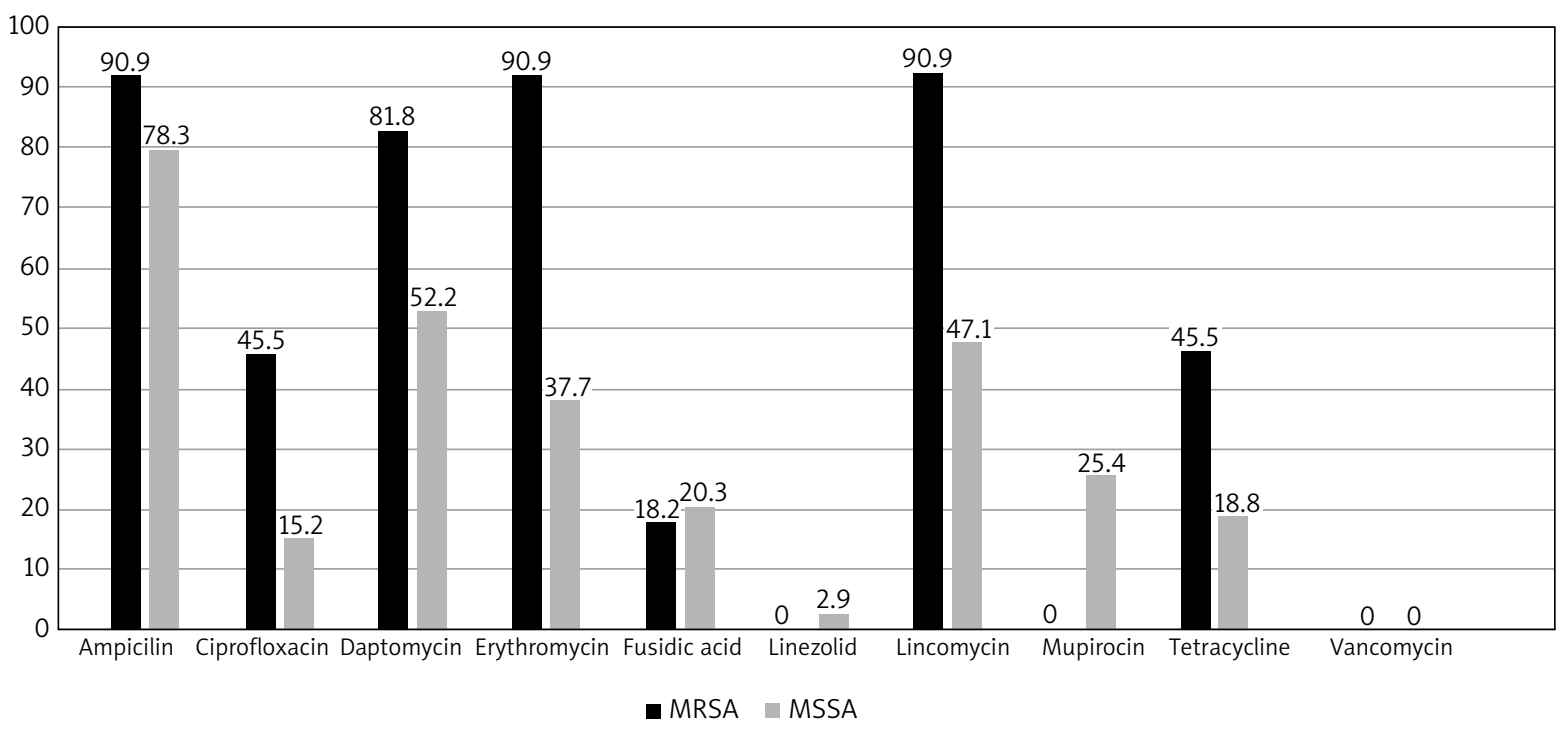

Figure 2. Antimicrobial resistance patterns (\%) of S. aureus in patients with atopic dermatitis among MRSA and MSSA strains

Zealand, $2 \%$ of $S$. aureus isolates from pediatric $A D$ cases were MRSA [13]. In addition, Niebuhr et al. found MRSA in $3 \%$ of $S$. aureus isolates in patients with AD [14]. Up to $30 \%$ of S. aureus isolates from AD cases were reported to be MRSA in a Taiwanese study population in 2011 [15]. It was also reported that S. aureus colonies on the skin of patients with $A D$ easily changed from being methicillin sensitive to being methicillin resistant when antibacterial agents were administered over a long period of time [16]. Staphylococcus aureus strains were isolated from the majority of our patients, either from the skin (75\%) or the anterior nares (73\%). There are no prevalence data for MRSA colonization among AD patients in Poland. In the present study of the 100 nasal swabs, 6 (6\%) and 5 of 100 skin swabs (5\%) were positive for MRSA (54.5\% CA-MRSA, 45.5\% HA-MRSA). The definition of CA-MRSA was coined by the Center for Disease Control and Prevention (CDC) in 2000. It refers to MRSA infection in a person who has none of the following established risk factors: isolation of MRSA more than $48 \mathrm{~h}$ after hospital admission; history of hospitalization, surgery, dialysis or residence in a long-term care facility 
within one year of the MRSA culture date; the presence of an indwelling catheter or a percutaneous device at the time of culture; or previous isolation of MRSA [17]. More recently, the emergence of MRSA clone colonizing farm animals (livestock-associated (LA)-MRSA) have been reported in Europe [18]. LA-MRSA possesses the same virulence potential as $S$. aureus from humans and is associated with the same manifold clinical pictures. LA-MRSA represents about $13 \%$ of MRSA-linked severe skin and soft tissue infections [19]. It can enter hospitals either via patients who suffer from infections caused by these bacteria or via patients with nasal colonization. Finally, it can lead to nosocomial infections such as surgical site infections, infections after joint arthroplasty, ventilator associated pneumonia, or septicemia [20]. Usually LA-MRSA is resistant to $\beta$-lactams, macrolides, lincosamides, streptogramins, and tetracyclines. It is susceptible to glycopeptides, daptomycin, tigecycline, rifampicin, fusidic acid, fosfomycin, and linezolid.

\section{Antibiotic resistance}

The higher rate of MRSA in the AD population may reflect higher overall prevalence rates and different management strategies, such as antibiotic use. Therefore, they should be used only when clinical signs of infection are presented, rather than to decolonize. Several studies concerning the effect of antimicrobial treatment on the $S$. aureus colonization and the severity of inflammation gave conflicting results. In open or double-blind placebo-controlled trials, topical or systemic antibiotics were able to reduce colonization density and led to a partial improvement of skin lesions [21-23]. In addition, some authors reported that topical steroids in combination with antibiotic treatment can cause elimination of S. aureus from the skin in some patients with AD [24]. On the other hand, treatment with oral antibiotics did not lead to a significant improvement of $A D$ in two double-blind placebo-controlled studies $[25,26]$. There are reports suggesting that treatment with antibiotics can temporarily reduce bacterial colonization and improve $A D$ severity. However, these benefits last no longer than 3 months [27]. Antibacterials effective against S. aureus include azithromycin, cefuroxime axetil, clarithromycin and erythromycin, while MRSA eradication by clindamycin, fusidic acid, trimethoprim-sulfamethoxazole and intranasal mupirocin can be effective [28]. In our study, a high resistance rate to conventional antibiotics was noticed. Thirty-one percent of isolated strains were resistant to erythromycin and $15.5 \%$ to tetracycline. Our results are comparable with those of Kędzierska and Hoeger $[12,29]$. In those studies, the resistance rate to erythromycin was $14 \%$ and $18 \%$, respectively. The increasing resistance to macrolides may suggest that erythromycin should no longer be applied in this case. In the present study, strains resistant to fusidic acid (FA) and mupirocin were also noticed.

\section{Fusidic acid}

Fusidic acid is an antibiotic that has been used in clinical practice in Europe since the 1960s in the treatment of skin and soft tissue infections, including infected atopic dermatitis. Fusidic acid has a high in vitro activity against $S$. aureus, S. epidermidis, some Corynebacterium and Gram-positive anaerobes such as Clostridium. Fusidic acid-resistant S. aureus (FRSA) has been reported in many countries, with the prevalence ranging from $0.3 \%$ to $52.5 \%[30,31]$. Accordingly, in the present study, $15.5 \%$ of strains resistant to fusidic acid were noted. Staphylococcus aureus resistance to FA is known to be already higher in dermatology patients compared with other disciplines and may result in treatment failure. Heng et al. reported FA resistance among $S$. aureus strains to be $11.3 \%$ among dermatology inpatients, which was higher than in patients of all disciplines (6\%) [32]. This higher rate of resistance is consistent with the findings by Shah and Mohanraj in the UK, with reported resistance rates of 50\% among dermatology outpatients, 9\% among primary care non-dermatology patients, and $10 \%$ among hospital inpatients [33, 34]. Katopodis et al. reported extremely high rates of FA resistance (88.9\%) among children in Greece with CAMRSA infections and acknowledged widespread use of FA in the community [35]. In contrast, in China where topical FA has been available only since 2003, Liu et al. detected no FA resistance in children with CA-MRSA infection [36]. Two major fusidic acid resistance mechanisms have been reported in S. aureus namely, the alteration of the drug target site which is due to mutations in fusA (encoding elongation factor $G-E F-G$ ) or rplF (FusE, encoding ribosome protein L6) [37] and the protection of the drug target site by FusB family proteins, including fusB, fusC and fusD [38]. Inappropriate use of topical antibiotics leading to resistance may threaten the efficacy of systemic antibiotics for the treatment of serious $S$. aureus infections such as osteomyelitis and severe surgical wound infections. Therefore, the topical use of FA for empirical treatment and decolonization of S. aureus should be restricted. Limiting the use of FA to less than two weeks for mild and localized secondary infection in atopic eczema has been recommended by the national guidelines [39].

\section{Mupirocin}

Mupirocin (pseudomonic acid A) is approved in ointment formulation in the United States for the topical treatment of impetigo and secondary wound infection caused by S. aureus and S. pyogenes. In addition, a nasal formulation is approved by the United States Food and Drug Administration (FDA) for use in eradicating nasal carriage of $S$. aureus in adult patients and health care personnel, as part of a comprehensive infection control program to reduce the risk of infection among patients 
at high risk of MRSA. Mupirocin is also increasingly used outside the hospital context because of the growing incidence of CA-MRSA and because, to date, mupirocin resistance levels have been lower than those of hospital-acquired MRSA [40]. Nevertheless, the increasing resistance to this agent has been reported and three categories of susceptibility for $S$. aureus were described. These categories are: mupirocin susceptibility with MICs below $1 \mu \mathrm{g} / \mathrm{ml}$, a low-level mupirocin resistance with MICs from 8 to $64 \mu \mathrm{g} / \mathrm{ml}$, and a high-level mupirocin resistance with MICs over $512 \mu \mathrm{g} / \mathrm{ml}$ [41]. In the present study, the low-level mupirocin resistance was noted in $9.46 \%$ and high-level one in $16.89 \%$ of the isolated strains. Most isolates that demonstrate high-level mupirocin resistance have acquired plasmid-mediated mupA, which encodes a novel isoleucyl RNA synthetase [42]. Isolates with low-level mupirocin resistance usually have acquired base changes in the native isoleucyl RNA synthetase gene, ileS [40]. Controlling MRSA transmission and infections is important in healthcare facilities, and decolonization is often recommended to achieve this goal (strength of evidence, IB-II) [43]. However, many reports emphasize the need to exercise caution when using this strategy, because carriage of MRSA with lowlevel mupirocin resistance is strongly associated with persistent colonization after eradication therapy [44]. If the clinical use of mupirocin increases, an increase in the prevalence of mupirocin resistance is likely to follow.

\section{Antiseptic agents}

Antiseptic agents represent an alternative to topical antibiotics in patients with $A D$, which are not generally recommended in the treatment of $A D$. An exception to the above antimicrobial agents is the use of bleach baths with intranasal topical mupirocin. According to Huang et al., chronic use of dilute bleach baths with intermittent intranasal application of mupirocin ointment decreased the clinical severity of $A D$ in patients with clinical signs of secondary bacterial infections [45]. Except for bleach baths with intranasal mupirocin, no topical antistaphylococcal treatment has been shown to be clinically helpful in patients with $A D$, and is not routinely used [46]. Antiseptics have been found to be better tolerated and less likely to induce bacterial resistance as compared to antibiotics. The main advantages of antiseptics over antibiotics are that they have low potential of inducing bacterial resistance, rarely cause delayedtype hypersensitivities or allergic reactions and come in a variety of preparations to suit individual needs [47]. Besides bleach baths, other antiseptics (triclosan, potassium permanganate, chlorhexidine gluconate and polyhexanide) are also available as an alternative, but at higher cost and without any randomized controlled trials published to date [48].

\section{Conclusions}

There are no prevalence data for MRSA colonization among AD patients in Poland. The aim of this study was to determine the prevalence of MRSA and S. aureus colonization in this group of patients, and to evaluate the antibiotic resistance of the isolated strains, especially fusidic acid and mupirocin, which are widely used for decolonization of S. aureus. In the present study, 10\% of isolations represented methicillin-resistant S. aureus. Among the tested antibiotics, $17.5 \%$ of strains were resistant to mupirocin and $15.5 \%$ to FA. The authors suggest that topical fusidic acid and mupirocin should be used to treat acute skin infections only for a short period of time. Furthermore, topical therapy of bacterial skin infections promotes the development of resistant strains. We hope that the cautious use of these topical antimicrobials will reverse the trend of increasing resistance observed among healthy subjects with colonization of S. aureus. The current popular practice of using diluted bleach baths once or twice weekly to reduce cutaneous staphylococcal colonization may also in the future alter the prevalence of MRSA and $S$. aureus in AD patients. However, there are many limitations to these studies, which therefore warrant further investigation on the impact of antiseptic use in AD. The approach of adding antiseptic agents to emollients and body washes is a convenient measure of introducing an additional agent into the AD management regimen. Finally, this study clearly emphasizes that susceptibility tests of clinical $S$. aureus isolates to antimicrobial drugs should be regularly performed before initiating therapy. Moreover, due to the increased risk of bacterial resistance that may occur as a result of the habit of using antibiotics in AD too often, it is important to monitor the rate of resistance to oral or topical antistaphylococcal drugs.

\section{Conflict of interest}

The authors declare no conflict of interest.

\section{References}

1. Sidbury R, Davis DM, Cohen DE. Guidelines of care for the management of atopic dermatitis. J Am Acad Dermatol 2014; 71: 327-49.

2. Petry V, Bessa GR, Poziomczyck CS, et al. Bacterial skin colonization and infections in patients with atopic dermatitis. An Bras Dermatol 2012; 87: 729-34.

3. Gong JQ, Lin L, Lin T, et al. Skin colonization by Staphylococcus aureus in patients with eczema and atopic dermatitis and relevant combined topical therapy: a double-blind multicentre randomized controlled trial. Br I Dermatol 2006; 155: 680-7.

4. Zollner TM, Wichelhaus TA, Hartung A, et al. Colonization with superantigen-producing Staphylococcus aureus is associated with increased severity of atopic dermatitis. Clin Exp Allergy 2000; 30: 994-1000. 
5. Strange P, Skov L, Lisby S, et al. Staphylococcal enterotoxin B applied on intact normal and intact atopic skin induces dermatitis. Arch Dermatol 1996; 132: 27-33.

6. Balma-Mena A, Lara-Corrales I, Zeller J, et al. Colonization with community-acquired methicillin-resistant Staphylococcus aureus in children with atopic dermatitis: a crosssectional study. Int J Dermatol 2011; 50: 682-8.

7. Gomes PL, Malavige GN, Fernando N, et al. Characteristics of Staphylococcus aureus colonization in patients with atopic dermatitis in Sri Lanka. Clin Exp Dermatol 2011; 36: 195-200.

8. Matiz C, Tom WL, Eichenfield LF, et al. Children with atopic dermatitis appear less likely to be infected with community acquired methicillin-resistant Staphylococcus aureus: the San Diego experience. Pediatr Dermatol 2011; 28: 6-11.

9. Chung HJ, Jeon HS, Sung H, et al. Epidemiological characteristics of methicillin-resistant Staphylococcus aureus isolates from children with eczematous atopic dermatitis lesions. J Clin Microbiol 2008; 46: 991-5.

10. Hanifin JM, Rajka G. Diagnostic features of atopic dermatitis. Acta Derm Venereol Suppl (Stockh) 1980; 92: 44-7.

11. Wayne PA, USA: Clinical and Laboratory Standards Institute (CLSI). M100-S21: Performance standards for antimicrobial susceptibility testing. 2012; 32. 21 $1^{\text {st }}$ Informational Supplement.

12. Hoeger PH. Antimicrobial susceptibility of skin-colonizing S. aureus strains in children with atopic dermatitis. Pediatr Allergy Immunol 2004; 15: 474-7.

13. Hill SE, Yung A, Rademaker M. Prevalence of Staphylococcus aureus and antibiotic resistance in children with atopic dermatitis: a New Zealand experience. Australas J Dermatol 2011; 52: 27-31.

14. Niebuhr M, Mai U, Kapp A, et al. Antibiotic treatment of cutaneous infections with Staphylococcus aureus in patients with atopic dermatitis: current antimicrobial resistances and susceptibilities. Exp Dermatol 2008; 17: 953-57.

15. Tang CS, Wang CC, Huang CF, et al. Antimicrobial susceptibility of Staphylococcus aureus in children with atopic dermatitis. Pediatr Int 2011; 53: 363-7.

16. Akiyama H, Yeda M, Toi Y. Comparison of the severity of atopic dermatitis lesions and the density of Staphylococcus aureus on the lesions after antistaphylococcal treatment. J Infect Chemother 1996; 2: 70-4.

17. Morrison MA, Hageman JC, Klevens RM. Case definition for community-associated methicillin-resistant Staphylococcus aureus. J Hosp Infect 2006; 62: 241.

18. van Cleef BA, Monnet DL, Voss A, et al. Livestock-associated methicillin-resistant Staphylococcus aureus in humans, Europe. Emerg Infect Dis 2011, 17: 502-5.

19. Layer F, Cuny C, Strommenger B, et al. Current data and trends on methicillin-resistant Staphylococcus aureus (MRSA). Bundesgesundheitsblatt Gesundheitsforschung Gesundheitsschutz 2012; 55: 1377-86.

20. Witte W, Strommenger B, Stanek C, et al. Methicillin-resistant Staphylococcus aureus ST398 in humans and animals, Central Europe. Emerg Infect Dis 2007; 13: 255-8.

21. Brockow K, Grabenhorst P, Abeck D, et al. Effect of gentian violet, corticosteroid and tar preparations in Staphylococcus aureus-colonized atopic eczema. Dermatology 1999; 199: 231-6.

22. Luber H, Amornsiripanitch S, Lucky AW. Mupirocin and the eradication of Staphylococcus aureus in atopic dermatitis. Arch Dermatol 1988; 124: 853-4.
23. White MI, Nobel WC. Consequences of colonization and infection by Staphylococcus aureus in atopic dermatitis. Clin Exp Dermatol 1986; 11: 34-40.

24. Dhar S. Should topical antibacterials be routinely combined with topical steroids in the treatment of atopic dermatitis? Indian J Dermatol Venereol Leprol 2005; 71: 71-2.

25. Boguniewicz M, Sampson H, Leung SB, et al. Effects of cefuroxime axetil on Staphylococcus aureus colonization and superantigen production in atopic dermatitis. J Allergy Clin Immunol 2001; 108: 651-2.

26. Ewing $\mathrm{Cl}$, Ashcroft C, Gibbs AC, et al. Flucloxacillin in the treatment of atopic dermatitis. Br J Dermatol 1998; 138: 1022-9.

27. Gilani SJ, Gonzalez M, Hussain I, et al. Staphylococcus aureus re-colonization in atopic dermatitis: beyond the skin. Clin Exp Dermatol 2005; 30: 10-3.

28. Cardona ID, Cho SH, Leung DY. Role of bacterial superantigens in atopic dermatitis : implications for future therapeutic strategies. Am J Clin Dermatol 2006; 7: 273-9.

29. Kedzierska A, Kapińska-Mrowiecka M, Czubak-Macugowska M, et al. Susceptibility testing and resistance phenotype detection in Staphylococcus aureus strains isolated from patients with atopic dermatitis, with apparent and recurrent skin colonization. Br J Dermatol 2008; 159: 1290-9.

30. Castanheira M, Watters AA, Bell JM, et al. Fusidic acid resistance rates and prevalence of resistance mechanisms among Staphylococcus spp. isolated in North America and Australia, 2007-2008. Antimicrob Agents Chemother 2010; 54: 3614-7.

31. Castanheira M, Watters AA, Mendes RE, et al. Occurrence and molecular characterization of fusidic acid resistance mechanisms among Staphylococcus spp. from European countries (2008). J Antimicrob Chemother 2010; 65: 1353-8.

32. Heng YK, Tan KT, Sen P, et al. Staphylococcus aureus and topical fusidic acid use: results of a clinical audit on antimicrobial resistance. Int J Dermatol 2013; 52: 876-81.

33. Shah M, Mohanraj M. High levels of fusidic acid-resistant Staphylococcus aureus in dermatology patients. Br J Dermatol 2003; 148: 1018-20.

34. Mitra A, Mohanraj M, Shah M. High levels of fusidic acidresistant Staphylococcus aureus despite restrictions on antibiotic use. Clin Exp Dermatol 2009; 34: 136-9.

35. Katopodis GD, Grivea IN, Tsantsaridou AJ, et al. Fusidic acid and clindamycin resistance in community-associated, methicillin-resistant Staphylococcus aureus infections in children of Central Greece. BMC Infect Dis 2010; 10: 351.

36. Liu Y, Kong F, Zhang X, et al. Antimicrobial susceptibility of Staphylococcus aureus isolated from children with impetigo in China from 2003 to 2007 shows community-associated methicillin-resistant Staphylococcus aureus to be uncommon and heterogeneous. Br J Dermatol 2009; 161: 1347-50.

37. Norström T, Lannergård J, Hughes D. Genetic and phenotypic identification of fusidic acid-resistant mutants with the small-colony-variant phenotype in Staphylococcus aureus. Antimicrob Agents Chemother 2007; 51: 4438-46.

38. O'Neill AJ, McLaws F, Kahlmeter G, et al. Genetic basis of resistance to fusidic acid in staphylococci. Antimicrob Agents Chemother 2007; 51: 1737-40.

39. Akdis CA, Akdis M, Bieber T, et al. European Academy of Allergology and Clinical Immunology/American Academy of Allergy, Asthma and Immunology. Diagnosis and treatment of atopic dermatitis in children and adults: European Academy of Allergology and Clinical Immunology/American Academy 
of Allergy, Asthma and Immunology/PRACTALL Consensus Report. J Allergy Clin Immunol 2006; 118: 152-69.

40. McConeghy KW, Mikolich DJ, LaPlante KL. Agents for the decolonization of methicillin-resistant Staphylococcus aureus. Pharmacotherapy 2009; 29: 263-80.

41. Eltringham I. Mupirocin resistance and methicillin-resistant Staphylococcus aureus (MRSA). J Hosp Infect 1997; 35: 1-8.

42. Udo EE, Jacob LE, Mathew B. Genetic analysis of methicillinresistant Staphylococcus aureus expressing high- and lowlevel mupirocin resistance. J Med Microbiol 2001; 50: 909-15.

43. Calfee DP, Salgado CD, Milstone AM, et al. Strategies to prevent methicillin-resistant Staphylococcus aureus transmission and infection in acute carehospitals: 2014 update. Infect Control Hosp Epidemiol 2014; 35 Suppl 2: S108-32.

44. Lee AS, Macedo-Vinas M, François P, et al. Impact of combined low-level mupirocin and genotypic chlorhexidine resistance on persistent methicillin-resistant Staphylococcus aureus carriage after decolonization therapy: a case-control study. Clin Infect Dis 2011; 52: 1422-30.

45. Huang JT, Abrams M, Tlougan B, et al. Treatment of Staphylococcus aureus colonization in atopic dermatitis decreases disease severity. Pediatrics 2009; 123: e808-14.

46. Eichenfield LF, Tom WL, Berger TG, et al. Guidelines of care for the management of atopic dermatitis: section 2. Management and treatment of atopic dermatitis with topical therapies. J Am Acad Dermatol 2014; 71: 116-32.

47. Lee M, Van Bever H. The role of antiseptic agents in atopic dermatitis. Asia Pac Allergy 2014; 4: 230-40.

48. Schnopp C, Ring J, Mempel M. The role of antibacterial therapy in atopic eczema. Expert Opin Pharmacother 2010; 11: 929-36. 